\title{
Weak versus strong no-slip boundary conditions for the Navier-Stokes equations
}

\author{
Qaisar Abbas and Jan Nordström
}

\section{Post Print}

N.B.: When citing this work, cite the original article.

Original Publication:

Qaisar Abbas and Jan Nordström, Weak versus strong no-slip boundary conditions for the Navier-Stokes equations, 2010, Engineering Applications of Computational Fluid Mechanics, (4), 29-38.

Copyright: Engineering Applications of Computational Fluid Mechanics

Postprint available at: Linköping University Electronic Press

http://urn.kb.se/resolve?urn=urn:nbn:se:liu:diva-68523 


\title{
WEAK VERSUS STRONG NO-SLIP BOUNDARY CONDITIONS FOR THE NAVIER-STOKES EQUATIONS
}

\author{
Qaisar Abbas* and Jan Nordström* ${ }^{*} \dagger$ \\ * Department of Information Technology, Scientific Computing Division, \\ Uppsala University, Box 337, SE-751 05 Uppsala, Sweden \\ E-Mail: qaisar.abbas@it.uu.se (Corresponding Author) \\ ${ }^{\dagger}$ Department of Aeronautics and Systems Integration, \\ The Swedish Defense Research Agency, SE-164 90 Stockholm, Sweden
}

\begin{abstract}
We investigate the accuracy aspects of weak boundary conditions for the Navier-Stokes equations. As a model problem, the linear advection-diffusion equation for a boundary layer problem is analyzed. The analysis shows that the weak boundary conditions are advantageous compared with the strong boundary conditions. We exemplify the analysis of the advectiondiffusion problem by doing Navier-Stokes calculations and show that most of the conclusions for the model problem hold also for that case.
\end{abstract}

Key words: Navier-Stokes, Weak Boundary Conditions, No-slip Boundary Conditions

\section{INTRODUCTION}

Accurate calculations of global coefficients, such as lift or drag, are very important for the aerodynamics design of aircrafts, space vehicles and railcars. These quantities depend on the accuracy of the scheme close to the solid boundaries in the flow field. High order finite difference methods (HOFDM) provide an efficient approach for such cases. The efficiency of HOFDM can be used either to increase the accuracy for a fixed number of mesh points or to reduce the computational cost for a given accuracy by reducing the number of mesh points [6]. The drawback with HOFDM is the complicated boundary treatment, required to get a stable method.

We have considered summation-by-parts (SBP) operators $[2,4,6,8,9,10]$, whose accuracy is now well established. These operators in combination with weak boundary procedures like the simultaneous approximation term (SAT) method [1] always lead to stability. On the other hand, there is no general procedure to obtain stability when using strong boundary conditions and high order difference schemes. It can be shown that strong boundary conditions may ruin the stability $[5,7]$, even for low order operators. If the strong solution is stable, then the weak and strong solutions are the same in the limit of very fine meshes.

In [11], a high order accurate finite difference scheme for the compressible Navier-Stokes equations which is stable due to the use of the weak boundary conditions and summation-byparts (SBP) operators was presented. The weak imposition of no-slip boundary conditions 
was considered in [12] and stability and accuracy were discussed. In this paper we extend this analysis and focus on the accuracy aspects of the no-slip weak boundary conditions, especially for coarse realistic meshes. We analyse the linear advection-diffusion equation and make comparison of the weak boundary conditions with the strong boundary conditions for coarse and fine meshes. We extend the analysis for the model problem to Navier-Stokes equations and make comparison of the results.

The rest of the paper will proceed as follows. In section 2 we give some concepts and basic definitions. In Section 3 we introduce our model problem and derive energy estimates for the semi-discrete case and find conditions under which the numerical scheme will be stable. In section 4 we perform a steady-state analysis of our model problem by solving exactly the second order difference equation for the weak and strong boundary conditions and prove the convergence of the weak solution to the strong solution. In section 5, computations and additional analysis are done for second and fourth order schemes. In section 6 , we consider the compressible Navier-Stokes equations for boundary layer flows and make numerical experiments. The numerically computed results are compared with the Blasius boundary solutions. A study of slip velocity is done in section 7. We summarize and draw conclusions in section 8. All operators are given in Appendix-1.

\section{DEFINITIONS}

To describe the numerical scheme, we need the following definitions. The domain $0 \leq x \leq 1$ is discretized using $N+1$ equidistant grid points, $x_{j}=j \Delta x, j=0,1, \ldots, N, \quad \Delta x=1 / N$. The numerical approximation at grid point $x_{j}$ is denoted $v_{j}$, and the discrete solution vector $v^{T}=\left[v_{0}, v_{1}, \ldots, v_{N}\right]$. The derivative $u_{x}$ is approximated with a finite difference approximation such that it satisfies the SBP property, i.e.

$$
u_{x} \approx P^{-1} Q v, \quad Q+Q^{T}=B=\operatorname{diag}(-1,0, \ldots, 0,1), \quad P=P^{T}>0 .
$$

The derivative $u_{x x}$ is approximated with a compact finite difference approximation which also satisfies the SBP property, for more details see $[4,6,8,9]$, i.e.

$$
u_{x x} \approx P^{-1}(-A+B S) v, \quad A+A^{T} \geq 0, \quad B S v=\left(-(D v)_{0}, 0, \ldots, 0,(D v)_{N}\right), \quad P=P^{T}>0
$$

We define an inner product and corresponding norm for discrete real-valued vector functions $u, v \in R^{n}$ by

$$
(u, v)_{P}=u^{T} P v, \quad\|v\|_{P}^{2}=v^{T} P v
$$

More details on all the operators defined in (1) - (3) is given in appendix-1.

\section{THE ADVECTION-DIFFUSION EQUATION}

Consider the following advection-diffusion problem in one space dimension, 


$$
\begin{aligned}
& u_{t}+a u_{x}=\varepsilon u_{x x}, \quad 0 \leq x \leq 1, \quad t \geq 0, \\
& u(0, t)=g_{0}, \quad u(1, t)=g_{N}, \quad u(x, 0)=f(x) .
\end{aligned}
$$

In (4) $a, \varepsilon>0$ and $\varepsilon<<a$. It can be shown that (4) is well-posed. For more details on the subject of well-posedness, see [2, 4, 6].

\subsection{The semi-discrete problem}

The semi-discrete approximation of (4) using SBP operators, including the weak implementation of the boundary conditions, can be written as

$$
\begin{gathered}
v_{t}+a P^{-1} Q v=\varepsilon P^{-1}(-A+B S) v+\beta_{0} P^{-1}\left(v_{0}-g_{0}\right) e_{0}+\beta_{1} P^{-1}\left(v_{N}-g_{N}\right) e_{N}, \\
v(0)=f
\end{gathered}
$$

where $e_{0}=[1,0, \ldots, 0]^{T}$, and $e_{N}=[0,0, \ldots, 1]^{T}$. The parameters $\beta_{0}$ and $\beta_{1}$ are known as the penalty coefficients. The energy method applied to (5) with $g_{0}=g_{N}=0$ leads to

$$
\begin{aligned}
& \frac{d}{d t}\|v\|_{P}^{2}=-2 \varepsilon v^{T} A v-2 \varepsilon v_{0}(D v)_{0}+2 \varepsilon v_{N}(D v)_{N}+\left(2 \beta_{0}+a\right) v_{0}^{2}+\left(2 \beta_{1}-a\right) v_{N}^{2} \\
& =-2 \varepsilon(S v)^{T} R(S v)+\left(\begin{array}{c}
v_{0} \\
(D v)_{0}
\end{array}\right)^{T}\left(\begin{array}{cc}
2 \beta_{0}+a & -\varepsilon \\
-\varepsilon & -2 \varepsilon \gamma_{0} \Delta x
\end{array}\right)\left(\begin{array}{c}
v_{0} \\
(D v)_{0}
\end{array}\right) \\
& +\left(\begin{array}{c}
v_{N} \\
(D v)_{N}
\end{array}\right)^{T}\left(\begin{array}{cc}
2 \beta_{1}-a & \varepsilon \\
\varepsilon & -2 \varepsilon \gamma_{N} \Delta x
\end{array}\right)\left(\begin{array}{c}
v_{N} \\
(D v)_{N}
\end{array}\right) .
\end{aligned}
$$

In (6) we have used a splitting of $A$ as $v^{T} A v=(S v)^{T} R(S v)+\gamma_{0} \Delta x(D v)_{0}^{2}+\gamma_{N} \Delta x(D v)_{N}^{2}$, see Appendix-1 for details on the matrices $A, S$ and $R$.

An energy estimate is obtained if $R+R^{T} \geq 0$ and the $2 \times 2$ matrices in (6) are negative semidefinite. With the choice

$$
\beta_{0}<-\frac{\varepsilon}{4 \gamma \Delta x}-\frac{a}{2}, \quad \beta_{1}=-\frac{\varepsilon \theta}{4 \gamma \Delta x}, \quad \theta \geq 1
$$

both the matrices in (6) are negative semi-definite. Also, for $0 \leq \gamma_{0}, \gamma_{N} \leq 1$, the matrix $R$ is positive semi-definite and hence (5) has an energy estimate. For more details on stability, see [4]. With strong boundary conditions, (5) is used at the inner points only (without the penalty terms).

\subsection{The exact continuous steady-state solution}

For equation (4), the exact steady-state solution with $g_{0}=1$ and $g_{N}=0$ is 


$$
u(x)=\left(e^{a / \varepsilon}-e^{a x / \varepsilon}\right) /\left(e^{a / \varepsilon}-1\right)
$$

In equation (8), $\varepsilon$ corresponds to the thickness of the boundary layer. Some solutions for $a=1$, and taking different values of $\varepsilon$ are shown in Figure 1 .

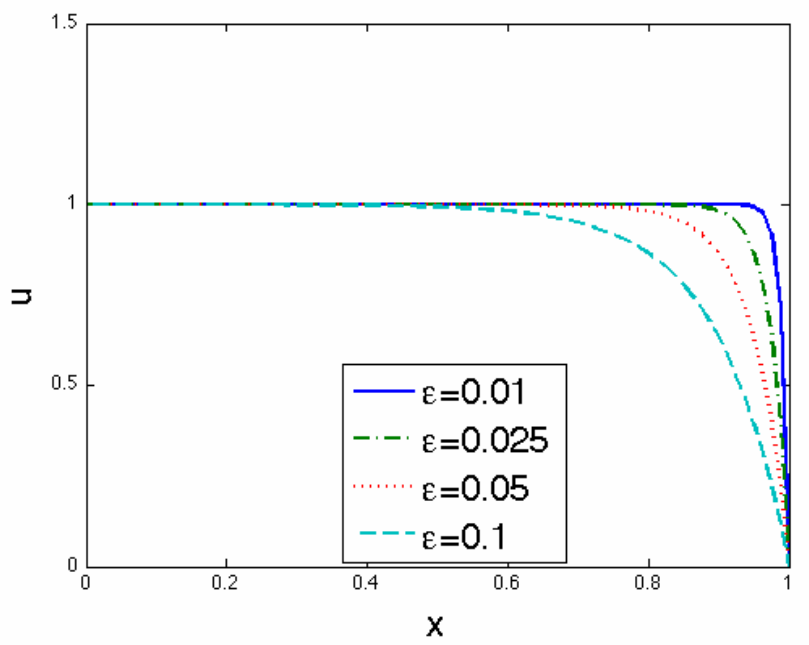

Figure 1: Steady-state solutions (An example of boundary layer)

\section{THE EXACT NUMERICAL STEADY-STATE SOLUTIONS}

The steady-state second order difference equation for our model problem (4) is given by

$$
a \frac{v_{i+1}-v_{i-1}}{2 \Delta x}=\varepsilon \frac{v_{i+1}-2 v_{i}+v_{i-1}}{(\Delta x)^{2}}
$$

Note that (9) corresponds to the steady-state version of (5) at inner points. The general solution to (9) is

$$
v_{i}=\alpha_{1}+\alpha_{2}\left(\frac{1+\alpha}{1-\alpha}\right)^{i}
$$

where $\alpha=a \Delta x / 2 \varepsilon$. The constants $\alpha_{1}, \alpha_{2}$ are determined by the boundary conditions.

From here onwards, the solution with weak boundary conditions will be denoted the weak solution and the solution with strong boundary conditions will be denoted the strong solution.

\subsection{The strong solution}

Applying strong boundary conditions $v_{0}=1, v_{N}=0$, and using (10) we get

$$
\alpha_{1}^{s}=-\left(\frac{1+\alpha}{1-\alpha}\right)^{N} /\left(1-\left(\frac{1+\alpha}{1-\alpha}\right)^{N}\right), \quad \alpha_{2}^{s}=1 /\left(1-\left(\frac{1+\alpha}{1-\alpha}\right)^{N}\right)
$$

The strong solution is given by inserting (11) into (10). 


\subsection{The weak solution}

The steady-state version of (5) at the boundary points $x_{0}$ and $x_{N}$ gives us (see Appendix-1)

$$
a \frac{v_{1}-v_{0}}{\Delta x}=\frac{2 \beta_{0}}{\Delta x}\left(v_{0}-1\right), \quad a \frac{v_{N}-v_{N-1}}{\Delta x}=\frac{2 \beta_{1}}{\Delta x}\left(v_{N}-0\right)
$$

Relations in (12) together with (10) give us the values for $\alpha_{1}^{w}, \alpha_{2}^{w}$. The result is

$$
\alpha_{1}^{w}=1-\alpha_{2}^{w}\left(1-\frac{a}{\beta_{0}} \frac{\alpha}{1-\alpha}\right), \quad \alpha_{2}^{w}=1 /\left[\left(1-\frac{a}{\beta_{0}} \frac{\alpha}{1-\alpha}\right)-\left(1-\frac{a}{\beta_{1}} \frac{\alpha}{1+\alpha}\right)\left(\frac{1+\alpha}{1-\alpha}\right)^{N}\right] .
$$

The weak solution is given by inserting (13) into (10).

\subsection{Relation between weak, strong and exact solutions}

Let $v^{w}$ and $v^{s}$ be the discrete steady-state solutions to problem (5) with weak and strong boundary conditions respectively. Let $u^{e}$ be the exact continuous solution to (4).

Theorem 1 If the mesh size goes to zero, i.e. $\Delta x \rightarrow 0$, then $v^{w} \rightarrow v^{s} \rightarrow u^{e}$.

Proof We know that the discrete strong and weak solutions are given by using (11), (13) and (10) respectively. By using the relation $\lim _{\Delta x \rightarrow 0}(1+\Delta x)^{1 / \Delta x}=e$, we get

$$
\lim _{\Delta x \rightarrow 0} \alpha_{1}^{w}=\alpha_{1}^{s}=1-\frac{1}{1-e^{a / \varepsilon}}, \quad \lim _{\Delta x \rightarrow 0} \alpha_{2}^{w}=\alpha_{2}^{s}=\frac{1}{1-e^{a / \varepsilon}}
$$

Relations (14) in combination with (8) and (10) prove the theorem.

Theorem 2 If $\beta_{0} \rightarrow-\infty, \beta_{1} \rightarrow-\infty$ in (5), then $v^{w} \rightarrow v^{s}$.

Proof In (7) if $\gamma \rightarrow 0$, we get $\beta_{0} \rightarrow-\infty, \beta_{1} \rightarrow-\infty$. That implies that

$$
\alpha_{1}^{w}=\alpha_{1}^{s}=-\left(\frac{1+\alpha}{1-\alpha}\right)^{N} /\left(1-\left(\frac{1+\alpha}{1-\alpha}\right)^{N}\right), \quad \alpha_{2}^{w}=\alpha_{2}^{s}=1 /\left(1-\left(\frac{1+\alpha}{1-\alpha}\right)^{N}\right)
$$

which proves the theorem.

We first investigate very coarse meshes and take $N=3,4$ and 5, and compare the weak, strong and exact solutions as given in Figure 2. Other parameters chosen are $a=1$ and $\varepsilon=0.1$. The weak solution is more accurate than the strong solution for all points except precisely at the boundary (where we know the solution anyway). This is true for all meshes with $\alpha<1$. 


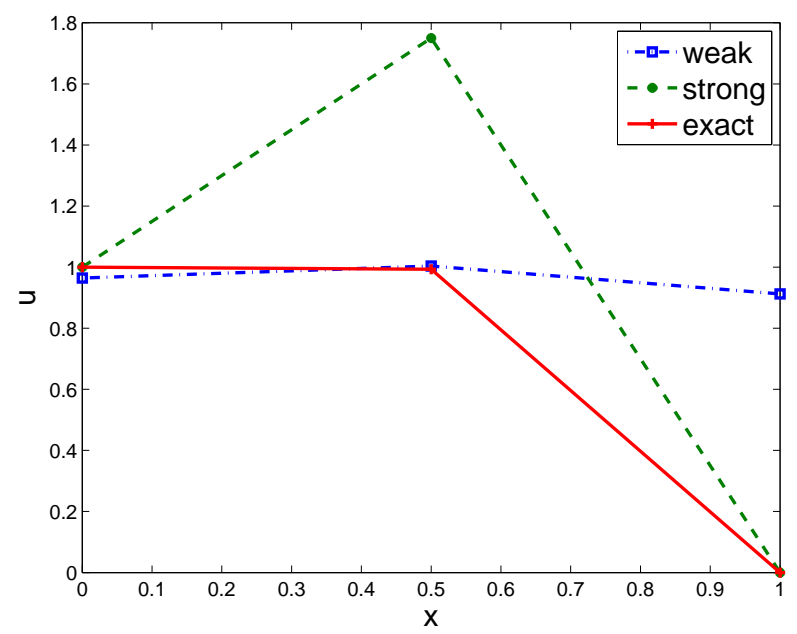

(a) $N=3$

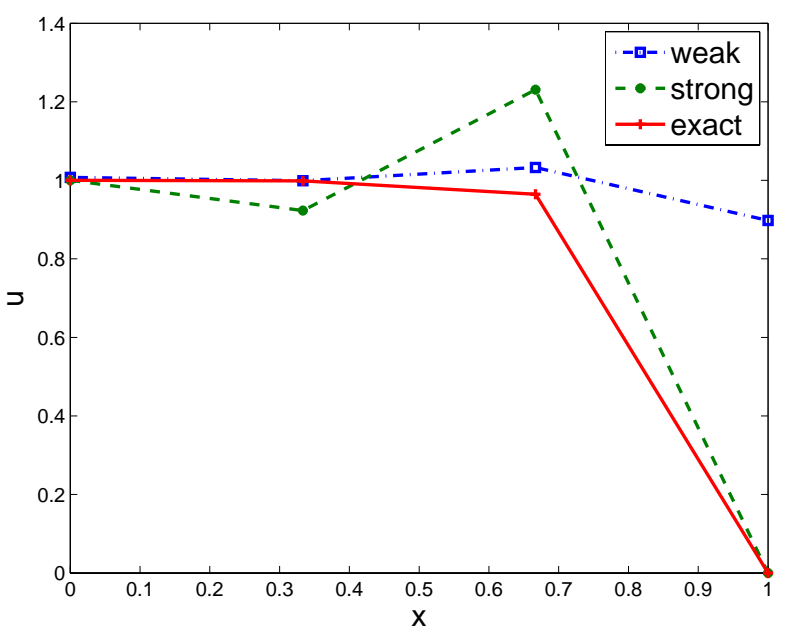

(b) $N=4$

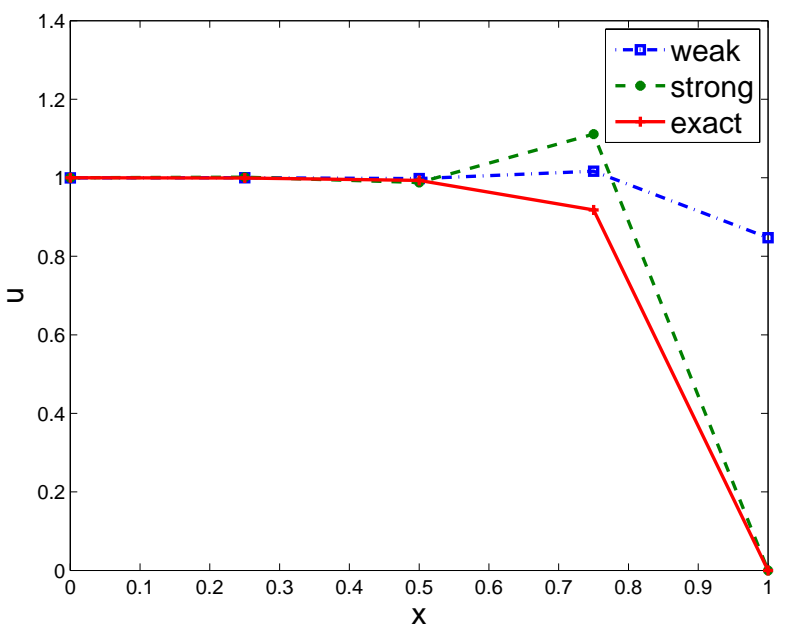

(c) $N=5$

Figure 2: The weak, strong and exact solutions for $a=1, \varepsilon=0.1$. 
Increased accuracy for the weak boundary conditions can be obtained by increasing the semibounded parameters $\beta_{0}$ and $\beta_{1}$. That can cause an increased stiffness that forces a reduced time step though. An alternative way is to refine the mesh or use stretching in the boundary layer region, as shown in graphs in Figure 3.

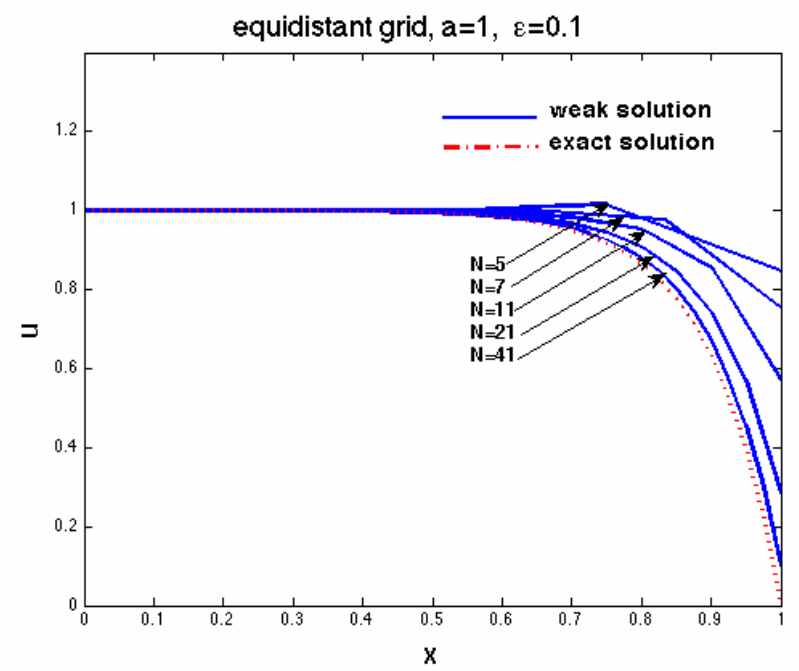

(a) Equidistant grid

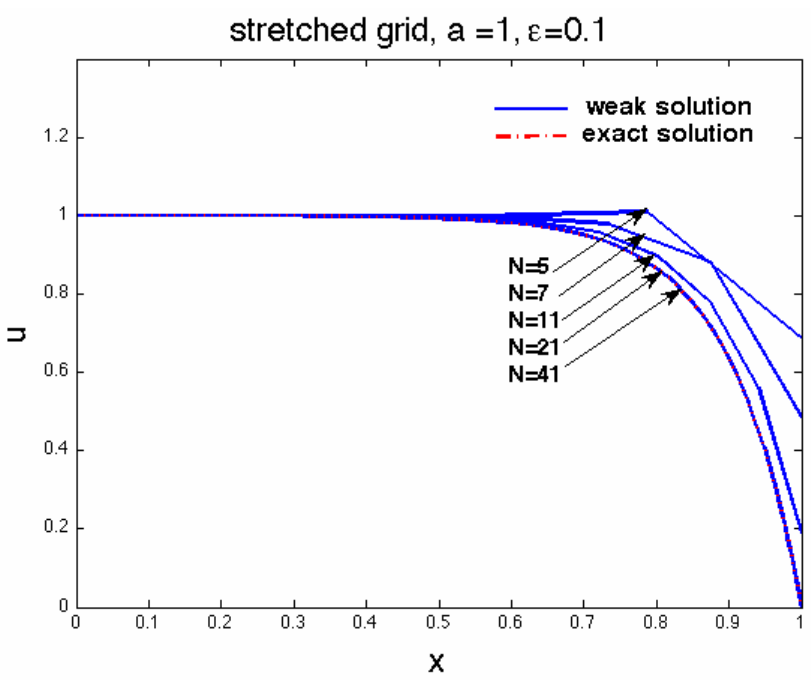

(b) Stretched grid

Figure 3: Convergence of the weak solution with mesh refinement

Note that the weak method gently forces the boundary condition from a (Euler) slip condition to a (Navier-Stokes) no-slip condition as the grid is refined, see also [3, 12].

\section{ACCURACY OF THE WEAK AND THE STRONG SOLUTIONS}

The convergence rate $q$ is defined as

$$
q=-\frac{\log _{10}\left(\left\|u-v^{(1)}\right\|_{2} /\left\|u-v^{(2)}\right\|_{2}\right)}{\log _{10}\left(N^{(1)} / N^{(2)}\right)},
$$


where $u$ is the exact steady-state solution given in (8). $v^{(1)}$ and $v^{(2)}$ are the corresponding numerical solutions with $N^{(1)}$ and $N^{(2)}$ grid points, respectively.

To verify the accuracy of (5), we do simulations with $a=1, \varepsilon=0.1$, while $\beta_{0}$ and $\beta_{1}$ are chosen on the stability limit such that (7) holds. We use the classical fourth-order RungeKutta method to integrate in time until we get a steady-state solution. We use the second- and the fourth-order schemes to compute the steady-state solutions and measure the convergence rates $q$ as given in Table 1. Note that the weak boundary conditions are more accurate for coarse meshes than the strong boundary conditions for the second order case. For the fourth order case, the strong boundary conditions are uniformly more accurate. The asymptotic convergence rates are the same.

Table 1: $\log _{10}\left(l_{2}-\right.$ error $)$, convergence rates at inner points

\begin{tabular}{|c|c|c|c|c|c|c|c|c|}
\hline \multirow{2}{*}{$\begin{array}{l}\text { Inner } \\
\text { points }\end{array}$} & \multicolumn{2}{|c|}{ Weak (2nd Order) } & \multicolumn{2}{|c|}{ Strong (2nd Order) } & \multicolumn{2}{|c|}{ Weak (4th Order) } & \multicolumn{2}{|c|}{ Strong (4th Order) } \\
\hline & $\log _{10}$ Err & $q$ & $\log _{10}$ Err & $q$ & $\log _{10}$ Err & $q$ & $\log _{10}$ Err & $q$ \\
\hline 1 & -1.99 & - & -0.12 & - & - & - & - & - \\
\hline 3 & -1.24 & -1.56 & -0.95 & 1.74 & - & - & - & - \\
\hline 7 & -1.08 & -0.43 & -1.61 & 1.80 & -1.56 & - & -1.95 & - \\
\hline 15 & -1.19 & 0.33 & -2.26 & 1.94 & -2.33 & 2.33 & -2.98 & 3.10 \\
\hline 31 & -1.54 & 1.10 & -2.88 & 1.96 & -3.33 & 3.17 & -4.13 & 3.65 \\
\hline 63 & -2.03 & 1.60 & -3.48 & 1.97 & -4.43 & 3.59 & -5.36 & 3.98 \\
\hline 127 & -2.59 & 1.83 & -4.09 & 1.98 & -5.59 & 3.79 & -6.61 & 4.12 \\
\hline 255 & -3.17 & 1.93 & -4.69 & 1.99 & -6.77 & 3.90 & -7.87 & 4.15 \\
\hline 511 & -3.77 & 1.97 & -5.29 & 1.99 & -7.96 & 3.95 & -9.12 & 4.13 \\
\hline
\end{tabular}

\section{THE COMPRESSIBLE NAVIER-STOKES EQUATIONS APPLIED TO BOUNDARY LAYER FLOWS}

We consider the flow on a flat plate of length $L$. The coordinate system is chosen such that $x$ is the streamwise coordinate along the plate and $y$ is the normal coordinate, perpendicular to the plate. The velocity components in the $x$ - and $y$-direction are denoted $u$ and $v$ respectively. A compressible steady-state solution with constant viscosity and thermal conductivity is considered. We study the development of the boundary layer on the plate subject to boundary data from the Blasius similarity solution as shown in Figure 4. A Mach number of 2 and a Reynolds's number of 10000 is used.
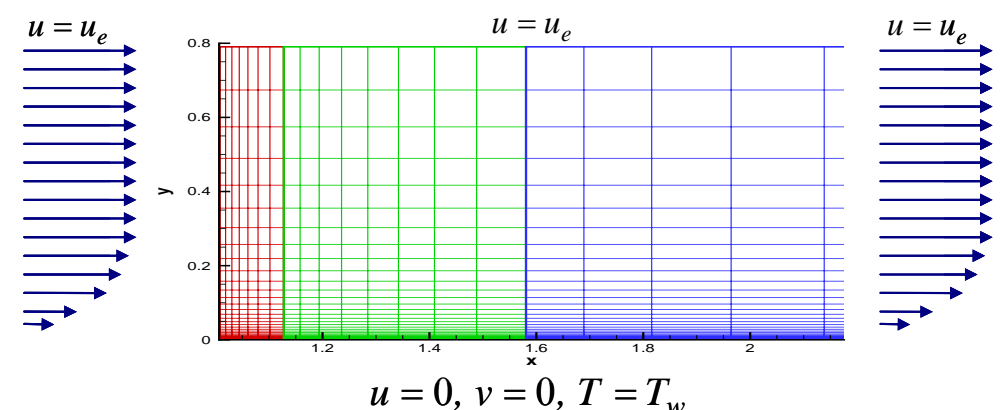

Figure 4: An example of stretched mesh for boundary layer calculations. 
We solve the compressible Navier-Stokes equations using second- and fourth-order schemes with weak and strong boundary conditions on different meshes. Stretched meshes are used both in $x$ - and $y$ - direction near the plate to capture the boundary layer features.
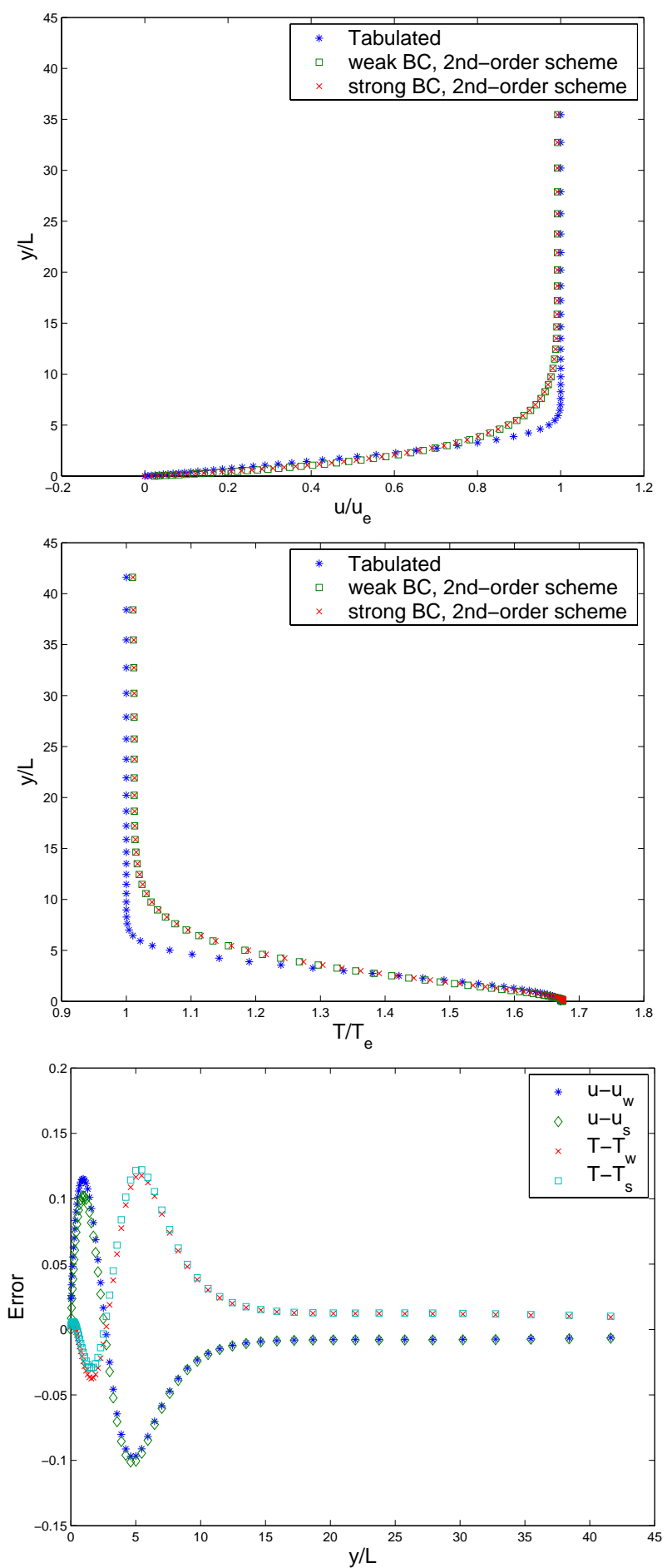

Figure 5: Navier-Stokes and similarity solutions, $2^{\text {nd }}$ order scheme, $\Delta x_{\min }=0.004, \Delta y_{\min }=0.0004$.

Two different meshes, one with $(\Delta y)_{\min }=0.0004$, and one with $(\Delta y)_{\min }=0.0001$ are used. We use weak boundary conditions on $u, v$ and $T$ in the weak case and implement strong boundary conditions on $u$ and $v$ while $T$ remains weakly imposed in the strong case. We 
integrate in time until we reach steady-state. The numerically computed solutions are compared to the available tabulated Blasius similarity solutions in Figure 5 to Figure 8.
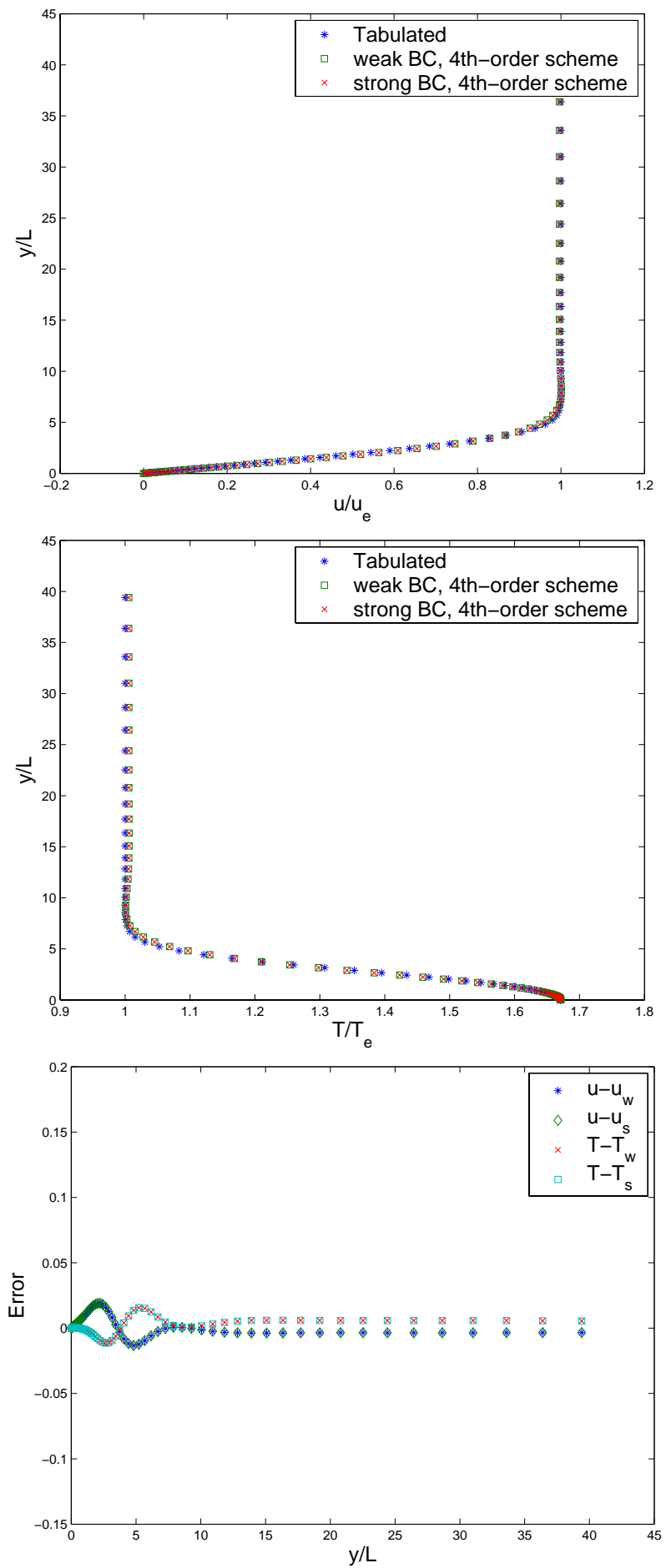

Figure 6: Navier-Stokes and similarity solutions, $4^{\text {th }}$ order scheme, $\Delta x_{\min }=0.004, \Delta y_{\min }=0.0004$.

In figure 5, we have shown normalized velocity $u / u_{e}$ and temperature $T / T_{e}$ and errors for a coarse mesh and second order scheme. The errors were obtained by subtracting from the Blasius solutions. It was found that the results closely follow the true (Blasius) solution. To see the accuracy of the scheme, we have computed solutions for a refined mesh in figure 7 and found that the errors decrease at the proper rate. An improved accuracy can also be obtained by using higher order scheme as shown in figures 6 and 8 . 
The quality of the calculations increases with increased number of mesh points and higher order of scheme. As expected the best results are obtained for the finest mesh and a fourth order scheme, see Figure 8.
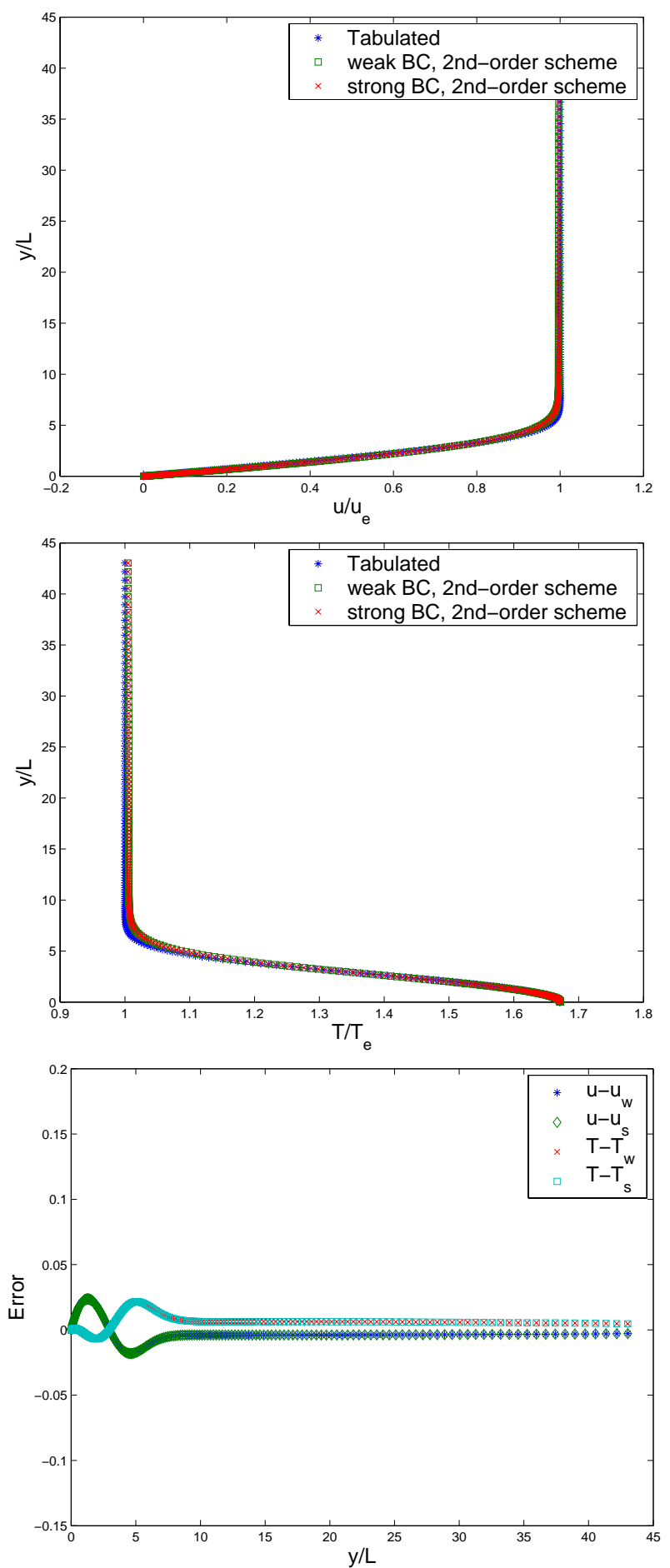

Figure 7: Navier-Stokes and similarity solutions, $2^{\text {nd }}$ order scheme, $\Delta x_{\min }=0.001, \Delta y_{\min }=0.0001$. 

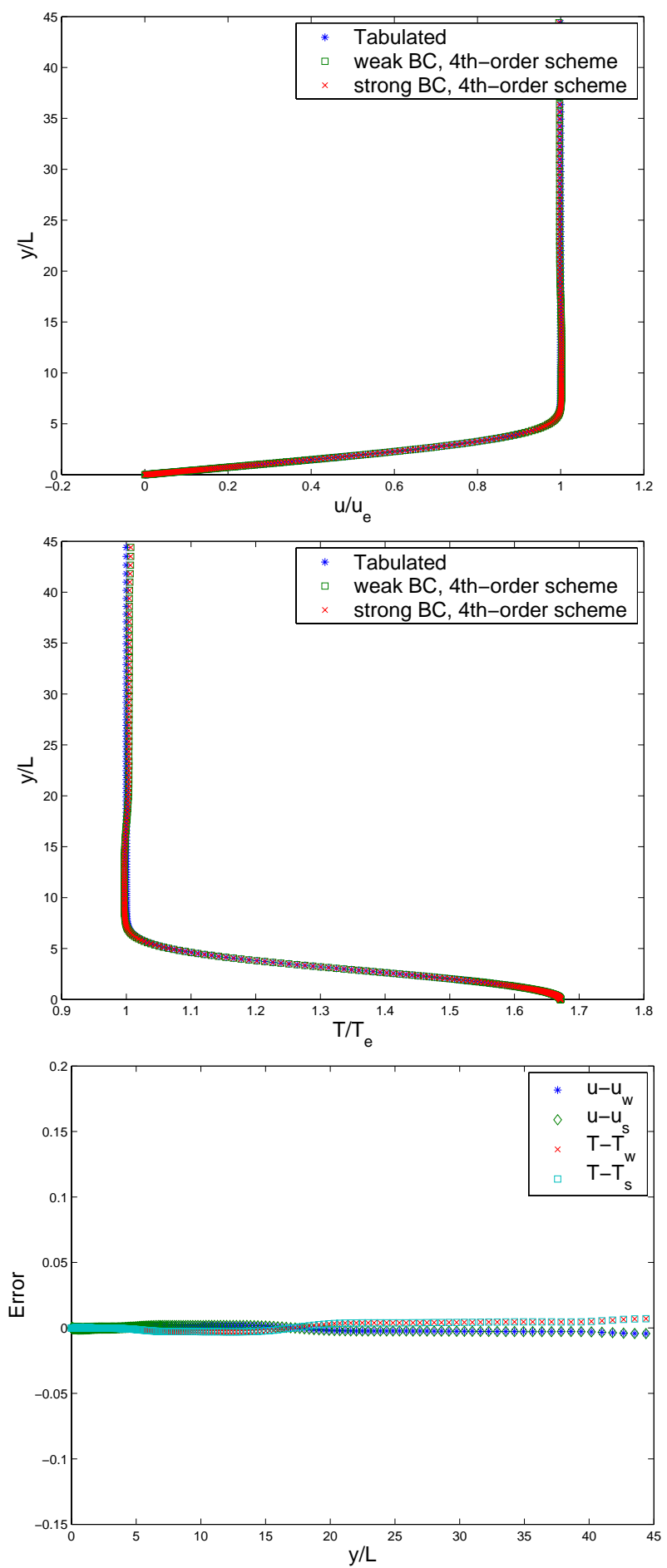

Figure 8: Navier-Stokes and similarity solutions, $4^{\text {th }}$ order scheme, $\Delta x_{\min }=0.001, \Delta y_{\min }=0.0001$.

\section{Study of slip velocity}

As we have already seen for the model problem, the weak solution behaves like an Euler solution for coarse meshes. Similar features could be observed for the Navier-Stokes equations see figure 9 . 


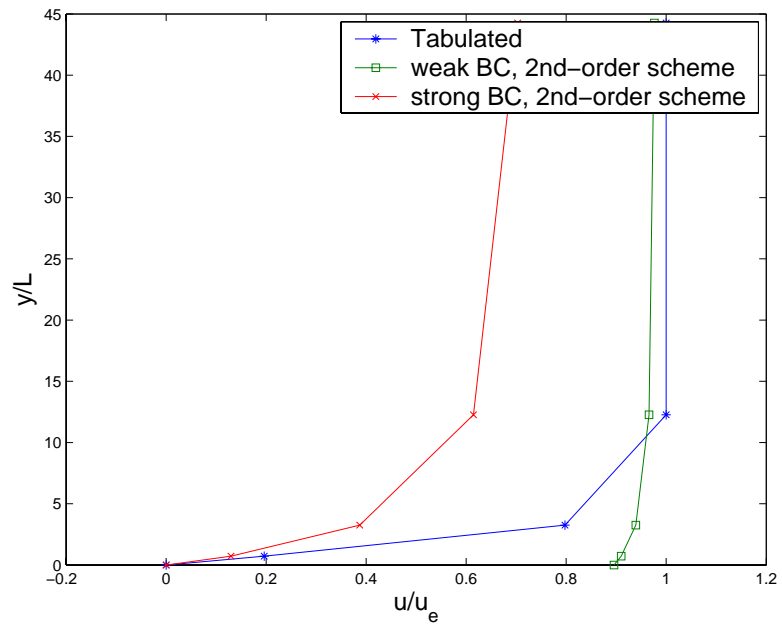

(a) $\Delta x_{\text {min }}=0.1, \Delta y_{\text {min }}=0.01$

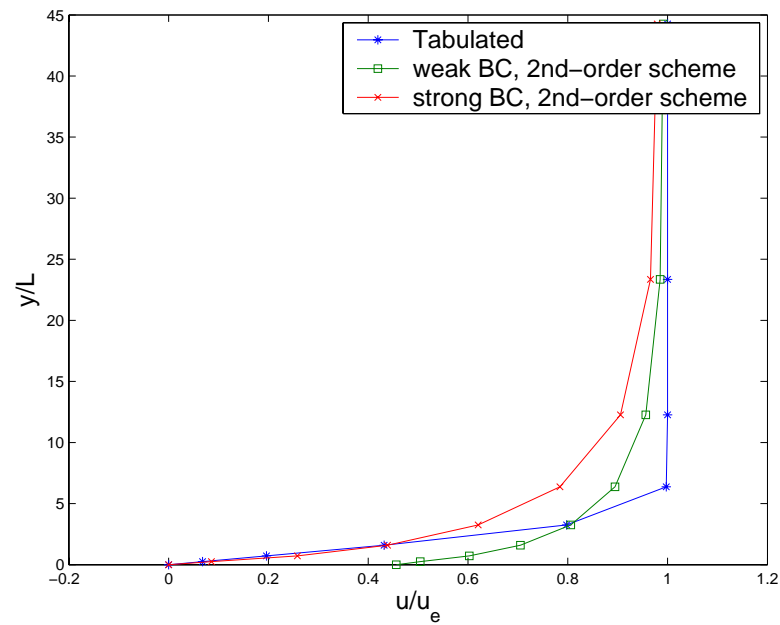

(b) $\Delta x_{\text {min }}=0.05, \Delta y_{\text {min }}=0.05$

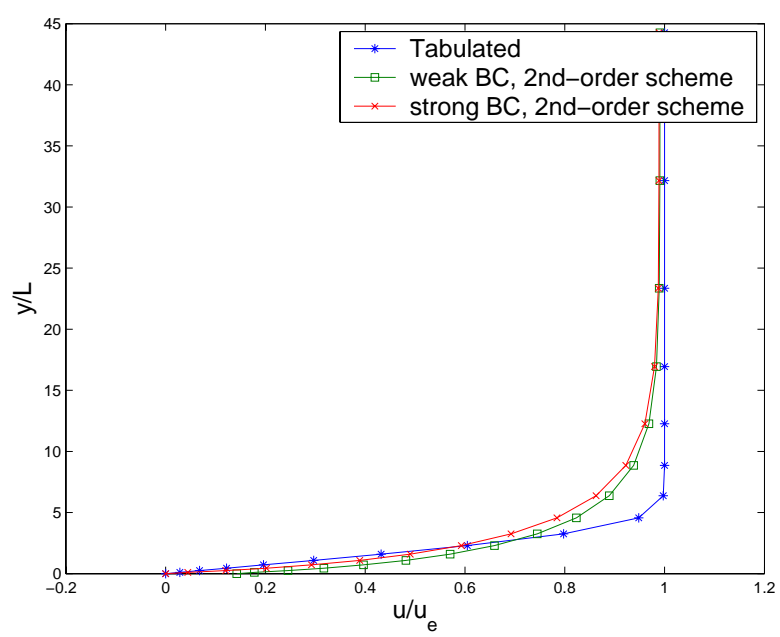

(c) $\Delta x_{\min }=0.02, \quad \Delta y_{\min }=0.002$

Figure 9: The weak boundary condition goes from a slip (Euler) to a no-slip (Navier-Stokes) condition. 
Moreover, the weak solution has the same asymptotic convergence rate as the strong solution for fine meshes. We notice that as the mesh is refined, the weak boundary conditions force the solution from a slip (Euler) condition to a no-slip (Navier-Stokes) condition.

\section{CONCLUSIONS}

Our main objective was to investigate the accuracy of HOFDM with weak and strong boundary conditions for boundary layer problems. We first considered a model problem and showed that in the limit of vanishing grid size or large penalty parameters, the weak solution converges to the strong solution.

We also showed that the weak solution is more accurate than the strong solution for coarse meshes in the second order case. We get a similar result for the full Navier-Stokes equations on coarse meshes, i.e. more accurate solutions are obtained with weak boundary conditions. On fine meshes, the strong solution is uniformly more accurate.

\section{Appendix-1}

The discrete second order accurate SBP operators $D_{1}=P^{-1} Q$ approximating $d / d x$ and the discrete norm $P$ are given by (17).

$$
D_{1}=\frac{1}{\Delta x}\left[\begin{array}{ccccc}
-1 & 1 & & & \\
-1 / 2 & 0 & 1 / 2 & & \\
& \ddots & \ddots & \ddots & \\
& & -1 / 2 & 0 & 1 / 2 \\
& & & -1 & 1
\end{array}\right], \quad P=\Delta x\left[\begin{array}{ccccc}
1 / 2 & & & & \\
& 1 & & & \\
& & \ddots & & \\
& & & 1 & \\
& & & 1 / 2
\end{array}\right]
$$

The discrete second order accurate SBP operator $D_{2}=P^{-1}(-A+B S)$ approximating $d^{2} / d x^{2}$ and the boundary derivative operator $B S$ are given by (18).

$$
D_{2}=\frac{1}{\Delta x^{2}}\left[\begin{array}{ccccc}
0 & 0 & 0 & & \\
1 & -2 & 1 & & \\
& \ddots & \ddots & \ddots & \\
& & 1 & -2 & 1 \\
& & 0 & 0 & 0
\end{array}\right], \quad B S=\frac{1}{\Delta x}\left[\begin{array}{ccccc}
1 & -1 & & & \\
& 0 & & & \\
& & \ddots & & \\
& & 0 & \\
& & -1 & 1
\end{array}\right]
$$

To get energy estimate in (7), we have splitted the matrix $A$ leading to

$$
A=S^{T}\left(R+\gamma_{0} E_{0}+\gamma_{N} E_{N}\right) S
$$

where $E_{0}$ and $E_{N}$ are zero matrices except $E_{0}(0,0)=E_{N}(N, N)=1$. The other matrices $A$ and $R$ in (18) are given by (20). 


$$
A=\frac{1}{\Delta x}\left[\begin{array}{ccccc}
1 & -1 & & & \\
-1 & 2 & -1 & & \\
& \ddots & \ddots & \ddots & \\
& & -1 & 2 & -1 \\
& & & -1 & 1
\end{array}\right], R=\Delta x\left[\begin{array}{ccccccc}
1-\gamma_{0} & & & & & & \\
& 1 & -1 & & & & \\
& -1 & 2 & -1 & & & \\
& & \ddots & \ddots & \ddots & & \\
& & & -1 & 2 & -1 & \\
& & & & -1 & 1 & \\
& & & & & & 1-\gamma_{N}
\end{array}\right] .
$$

\section{REFERENCES}

1. Carpenter MH, Gottlieb D, Abarbanel S (1994). Time-stable boundary conditions for finite-difference schemes solving hyperbolic systems: Methodology and applications to high-order compact schemes, J. Comput. Phys. 111:220- 236.

2. Carpenter MH, Nordström J, Gottlieb D (1999). A stable and conservative interface treatment of arbitrary spatial accuracy, J. Comput. Phys. 148:341-365.

3. Eriksson S, Svärd M, Nordström, J (2007). Simulations of ground effects on wake vortices at runways, Technical Report 2007-019, Department of Information Technology, Uppsala University, Sweden.

4. Gustafsson B, Kreiss HO, Oliger J (1995). Time dependent Problems and Difference Methods, Wiley-Interscience, New York.

5. Nordström J, Björck M (2001). Finite volume approximations and strict stability for hyperbolic problems, Applied Numerical Mathematics, 38, 237-255.

6. Nordström J, Carpenter MH (1999). Boundary and interface conditions for high order finite difference methods applied to the Euler and Navier-Stokes equations, J. Comput. Phys. 148:621-645.

7. Nordström J, Forsberg K, Adamsson C, Eliasson P (2003). Finite volume methods, unstructured meshes and strict stability for hyperbolic problems, Applied Numerical Mathematics, 45, 453-473.

8. Olsson P (1995). Summation by parts, projections, and stability, I. Math. Comp. 64 (211) 1035-1065.

9. Olsson P (1995). Summation by parts, projections, and stability, II, Math. Comp. 64 (212) $1473-1493$.

10. Strand B (1994). Summation by parts for finite difference approximations for $d / d x, J$. Comput. Phys. 110 (1) 47-67.

11. Svärd M, Carpenter MH, Nordström J (2007). A stable high-order finite difference scheme for the compressible Navier-Stokes equations, far-field boundary conditions, J. Comput. Phys. 225:1020-1038.

12. Svärd M, Nordström J (2008). A stable high-order finite difference scheme for the compressible Navier-Stokes equations: no-slip wall boundary conditions, J. Comput. Phys. 227:4805-4824. 


\section{Authors Addresses}

1.

\section{Qaisar Abbas}

PhD student

Address: Division of Scientific Computing Department of Information Technology

Uppsala University

Box 337

SE-751 05 Uppsala

Sweden

Phone: +46 18 - 4716253

Fax: +46 $18523049,+4618511925$

Email: qaisar.abbas@it.uu.se

2.

\section{Jan Nordström}

Adj. professor in numerical analysis

Address: Division of Scientific Computing

Department of Information Technology

Uppsala University

Box 337

SE-751 05 Uppsala

Sweden

Phone: +46 18 - 4712975

Fax: +4618523049, +4618511925

Email: Jan.Nordstrom@it.uu.se 